\title{
Bioaerosol characterization by surface-enhanced Raman spectroscopy (SERS)
}

\author{
A. Sengupta ${ }^{\mathrm{a}}$, M.L. Laucks ${ }^{\mathrm{b}}$, N. Dildine ${ }^{\mathrm{b}}$, E. Drapala ${ }^{\mathrm{b}}$, E.J. Davis $^{\mathrm{b}, *}$ \\ ${ }^{a}$ Department of Chemistry, University of Washington, Seattle, WA 98195-1700, USA \\ ${ }^{\mathrm{b}}$ Department of Chemical Engineering, University of Washington, Box 351750, Seattle, WA 98195-1750, USA
}

Received 30 April 2004; received in revised form 18 August 2004; accepted 4 November 2004

\begin{abstract}
It is demonstrated that bioaerosols can be characterized by their surface-enhanced Raman spectra. Preliminary studies showed that Raman spectra of aqueous suspensions of Escherichia coli, Pseudomonas aeruginosa, and Salmonella typhimurium can be obtained by mixing them with a nanocolloidal suspension of silver. Adsorption of the microorganisms on the silver quenches fluorescence and yields greatly enhanced spectra. To assist in the interpretation of the spectra we also obtained SERS spectra of $N$-acetyl glucosamine and the amino acids L-lysine, D-alanine and D-glutamic acid. After baseline studies identified the spectral region of interest and the concentrations of microorganisms and colloid particles needed to maximize the Raman signal, aerosolized microorganisms were impacted and transferred to a colloidal suspension of silver nanoparticles to obtain reproducible Raman spectra. (C) 2004 Elsevier Ltd. All rights reserved.
\end{abstract}

Keywords: Bioaerosols; E. coli bacteria; Pseudomonas; Salmonella; Surface-enhanced Raman spectroscopy

\section{Introduction}

The national concern over terrorism, and specifically bioterrorism, has greatly increased the interest in the detection and identification of bioaerosols. Although the mailing of envelopes containing anthrax spores (Bacillus anthracis) to politicians and journalists in October 2001 has stimulated much research to develop biosensors, there has long been an interest in bioaerosols as respiratory irritants for asthmatics and individuals who suffer from allergic reactions to fungi and mold.

\footnotetext{
* Corresponding author. Tel.: +1 206543 0298; fax: +1 2065433778.

E-mail address: davis@cheme.washington.edu (E.J. Davis).
} 
Conventional off-line methods of detecting bioaerosols by removing them from a surface (or filter) upon which they have been deposited and analyzing them by microscopic, chemical, and biological assays do not provide rapid identification and response. For example, in their studies of filters for bioaerosol collection, Wang, Reponen, Willeke, and Grinshpun (1999) and Wang, Reponen, Grinshpun, Górny, and Willeke (2001) eluted bacteria from the filters and incubated samples of $B$. subtilis for $18 \mathrm{~h}$, and $P$. fluorescens for 40-48 h. They then counted the colonies formed in suitable growth media.

Common aerosol sampling systems include impactors and filters, and a number of studies of bioaerosol sampling by such devices have been reported. These include an evaluation of the performance of AirO-Cell, Burkard, and Button samplers by Aizenberg, Reponen, Grinshpun, and Willeke (2000), a comparative study of the survival ratio of bacteria in aerobiological samplers by Henningson, Lundquist, Larsson, Sandstrom, and Forsman (1997), swirling airborne microorganism collection by Lin et al. (2000), collection of bioaerosols by electrostatic precipitation and the effect of field charge by Mainelis et al. (1999, 2001, 2002a,b) and Mainelis, Willeke, Adhikari, Reponen, and Grinshpun (2002) and sampling bioaerosols (E. coli and B. subtilis) with an impactor using a cooled impaction plate by Lee and Kim (2002). Stewart et al. (1995) examined the effect of impact stress on microbial recovery. If one is to use impactors or filters to trap bioaerosols, there is a need to identify microorganisms quickly and, preferably, on-line.

Alternate approaches to biodetection have been proposed and/or developed such as those at Lawrence Livermore National Laboratory (LLNL) for real-time sample collection, detection, and identification in the field (Walter, 1998). These include a miniature flow cytometer system (miniFlo) and a portable DNA analysis system using the polymerase chain reaction (PCR) to make copies of microbial or viral DNA that are subsequently identified by means of fluorescently labeled DNA designed to hybridize to signature DNA sequences of specific organisms. Additional work at LLNL on bioagent detection involves the use of dielectrophoresis for detecting bacterial cells and an acoustic concentrator for aerosol detection. PCR technology requires anticipation of the pathogens to be detected because appropriate specific fluorescence probes must be added to the bioagent. Similarly, detection of viruses and proteins using flow cytometry requires the addition of beads coated with an antibody. Despite these limitations, in field trials the miniFlo was effective in detecting Bacillus globigii and Erwinia herbicola (simulants for anthrax and plague, respectively).

Except for the field studies carried out by Lawrence Livermore National Laboratory, most of the published work on the identification of microorganisms has been limited to laboratory studies in which a surface has been pre-treated in some way to permit identification of a specific microorganism or in which the microorganism has been processed by washing, drying, etc.

Real-time detection of bioaerosols was proposed by Hill et al. (1995), Nachma et al. (1996), and Hairston, Ho, and Quant (1997) by measuring intrinsic fluorescence, but the natural fluorescence does not usually provide a sufficiently distinct spectral fingerprint to identify the bioagent (Laucks, Davis, Roll, \& Schweiger, 2000). The development of single aerosol particle time-of-flight mass spectrometers (ATFMS) has made it possible to perform rapid chemical analysis of inorganic and some organic particulate matter. The coupling of ATFMS with matrix assisted laser desorption/ionization (MALDI) looks promising for the on-line detection and identification of bioaerosols (Marijnissen, Van Wuijckhuijse, \& Stowers, 2003; Murray, Jackson, Mishra, \& Kim, 2003), but the extensive instrumentation required limits the widespread utilization of the technique.

In this paper we report on an approach to bioaerosol detection and characterization that combines aerosol technology, nanotechnology, and inelastic light scattering that may permit real-time or 
near-real-time identification of aerosol particles at relatively low cost, and, with some improvement, compact instrumentation.

\section{Surface-enhanced Raman spectroscopy}

Raman spectroscopy and resonance Raman spectroscopy (RRS) are important tools for the analysis and identification of biological species (e.g., see the monographs by Carey (1982) and Parker (1983)). Raman spectroscopy has been used extensively to study a wide range of viruses and biomolecules in aqueous solution, and new applications of Raman spectroscopy, such as an incisive probe for biomolecules, are frequently reported (e.g., Freemantle, 2003). Much less work has been devoted to the detection of airborne bioagents using inelastic light scattering techniques, except for fluorescence measurements.

The spontaneous Raman effect is so weak that fluorescence, when it occurs, obscures the Raman spectrum, and biomaterials generally contain fluorophores that exhibit strong fluorescence. Resonance Raman requires a tunable laser source to probe the various absorption bands in biomaterials. Surfaceenhanced Raman spectroscopy (SERS) suppresses fluorescence and greatly increases the Raman signal. Therefore, SERS appears to be a suitable probe for determining the chemical composition of biomaterials, especially when the detection of small amounts of material is required. We have studied surface-enhanced Raman scattering (SERS) as a method for distinguishing harmful bioaerosols from each other and from background biological material (pollen, non-pathogenic bacteria). It appears that SERS has a number of advantages over other methods of bioaerosol detection because it can be incorporated into a real-time and potentially portable detection system for micro-amounts of biomaterial.

Recently, Alexander, Pellegrinol, and Gillespie (2003) reported the use of near-infrared SERS to obtain spectra of single optically trapped bacterial spores in an aqueous medium. For the SERS substrate they used $\sim 60 \mathrm{~nm}$ diameter gold colloidal particles bound to 3-aminoproplytriethoxysilane-derivatized glass. Light from a $787 \mathrm{~nm}$ laser diode was used for the optical tweezers and to excite the SERS. They published spectra for two different strains of single Bacillus stearothermophilus spores and compared the SERS spectrum for a single spore with the spectrum for conventional Raman scattering from a single spore, finding a relatively modest enhancement of order $10^{2}$. Efrima and Bronk (1998) and Jarvis and Goodacre (2004) used silver colloids to characterize bacteria by SERS. These authors reported SERS measurements for aqueous solutions of bacteria, and Efrima and Bronk found that the SERS results for different types of bacteria are nearly indistinguishable. But Jarvis and Goodacre reported subtle quantitative differences in "raw" spectra of E. coli isolate and Enterococcus sp. (ENTC90) isolate.

In many of the experiments it is not clear how reproducible the results are-no information on the ratios of bacteria and colloid solutions used or how much the mixtures of one bacteria with one colloid solution varied. Most experiments have been done at a laser wavelength of $785 \mathrm{~nm}$. Although there is some evidence that use of near-IR wavelengths reduces the fluorescence background in SERS spectra, we obtained reproducible, low background spectra using a laser wavelength of $514 \mathrm{~nm}$.

One reason for choosing the visible excitation wavelength is that past investigations have revealed that a secondary extinction band appears at longer wavelengths from the original extinction band of the colloid after the addition of analyte. This secondary extinction band is an indicator that larger colloidal aggregates have been induced by the analyte. Creighton, Blatchford, and Albrecht (1979) demonstrated from UV studies of the colloidal systems that, for pyridine derivatives, the secondary extinction band of silver colloid appears in the region $525-550 \mathrm{~nm}$ after aggregation. They found that the Raman intensity was a 
maximum whenever the excitation wavelength was near the large particle excitation band. In this region the SERS signal is further strengthened by the strong resonance between the oscillation of free electrons in the metal and the electromagnetic radiation. For silver hydrosols the visible excitation wavelength is more or less near this ideal condition. Furthermore, at near-IR excitation wavelengths, there is a chance of thermal degradation of the biological sample on prolonged exposure to laser radiation. Sample degradation is less of a problem at visible excitation wavelengths.

In this paper we present SERS spectra from aerosolized bacteria. To our knowledge there is no such report of applying SERS to detect aerosolized bacteria. These studies have been motivated by a desire to understand whether common bacteria and pathogenic bacteria can be distinguished using Raman spectroscopy. We focused on SERS since there is evidence that SERS quenches fluorescence and provides the large signal enhancement necessary to detect small amounts of material.

\section{Apparatus}

An existing Raman facility was modified to pass a laser beam through an optical glass cuvette (interior dimensions: $1 \mathrm{~cm} \times 1 \mathrm{~cm} \times 4.5 \mathrm{~cm}$ ) containing a colloidal suspension of silver. The system is shown in Fig. 1. A $6 \mathrm{~W}$ argon-ion CW laser operating at a wavelength of $514.5 \mathrm{~nm}$ was the light source. Light scattered by the bioanalyte/silver colloid was focused on the slit of a $500 \mathrm{~mm}$ focal length single pass Acton SpectraPro-500I monochromator with a grating turret permitting dispersions of 300, 1200, and 2400 lines/mm. A Kaiser Optical Systems SuperNotch-Plus holographic filter was placed in front of the spectrometer to remove the laser line. The spectrum was recorded using a Princeton Instruments backilluminated, liquid nitrogen-cooled $1340 \times 100$ pixel array CCD camera. The laser beam power at the cuvette was typically $<250 \mathrm{~mW}$. The exposure time was typically $120 \mathrm{~s}$ except for the spectra of the aerosolized samples, which were integrated over $60 \mathrm{~s}$.

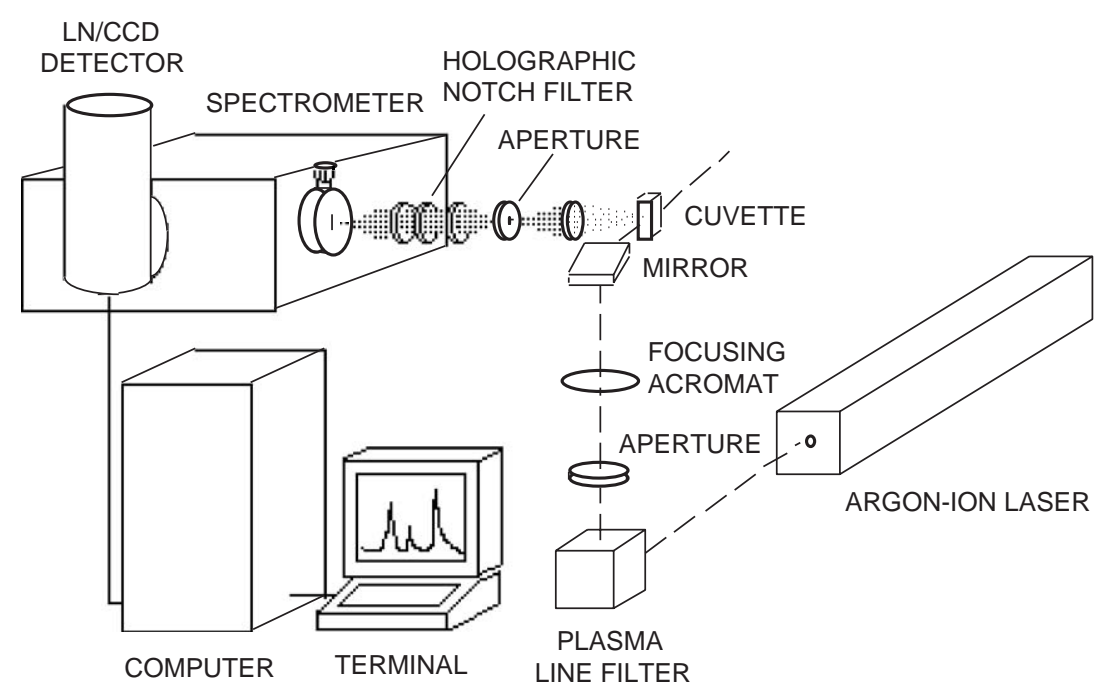

Fig. 1. The Raman system used for the SERS experiments. 


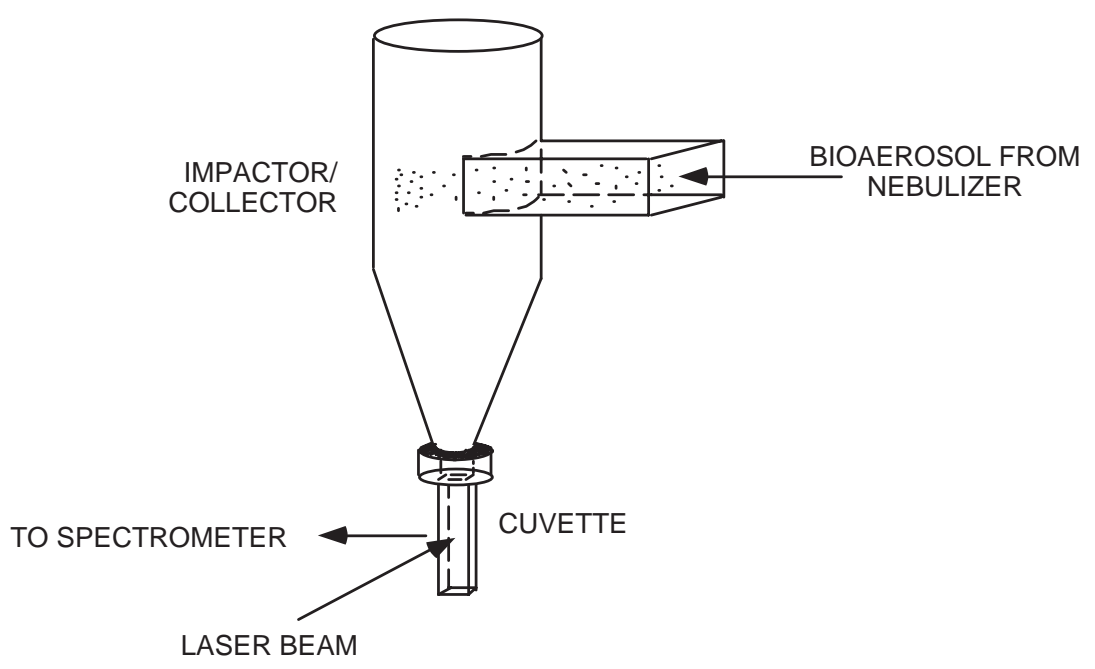

Fig. 2. The impactor/collector system used for sampling aerosolized bacteria.

After preliminary experiments established the desirable operating parameters, we carried out aerosol experiments in which an aqueous suspension of bacteria was nebulized, and the aerosol stream was impacted and collected in the device shown in Fig. 2. A commercial nebulizer (Onron NE-C21 compressor nebulizer system) was used to generate the aerosol from an aqueous suspension of the bacteria. The manufacturer reported an average aerodynamic diameter of $2.1 \mu \mathrm{m}$, but this was not verified in our experiments. The nebulizer was operated for $5 \mathrm{~min}$ to collect approximately 50-100 $\mu$ l of analyte. The aerosolized stream impacted the curved wall of the device shown and the analyte dripped down into the silver colloidal solution. By controlling the flow rate and collection time we could control the volume of the aerosolized sample introduced into the colloidal solution. In previous bioaerosol impaction studies mentioned above the collected material was analyzed off-line by conventional methods involving the growth of colonies over several hours in an appropriate growth medium.

\section{Experiments}

Initially, the bioanalyte was simply added to the colloid using a syringe in experiments designed to determine the appropriate spectral window, the desired laser irradiance, and the optimum ratio of the concentration of microorganisms to the colloid concentration.

We used silver colloid solutions (silver concentration $\sim 0.0002 \mathrm{M}$ ) to create the "surface" substrate for the SERS effect. The colloid was produced using the method of Keir, Sadler, and Smith (2002). Briefly, an aqueous solution of silver nitrate $(9 \mathrm{ml}, 0.0022 \mathrm{M})$ was added drop wise with continuous stirring to an aqueous sodium borohydride solution $(75 \mathrm{ml}, 0.0012 \mathrm{M})$ in a conical flask in an ice bath. The flask was finally removed from the ice bath and stirring was continued for a further $45 \mathrm{~min}$. We tested the $\mathrm{Ag}$ colloid solutions repeatedly for reproducibility and other characteristics. The colloid solutions are stable only for a period of days as indicated by their UV-VIS spectra. We determined a colloidal particle size using a scanning electron microscope. Fig. 3 is a representative SEM image showing that the mean 


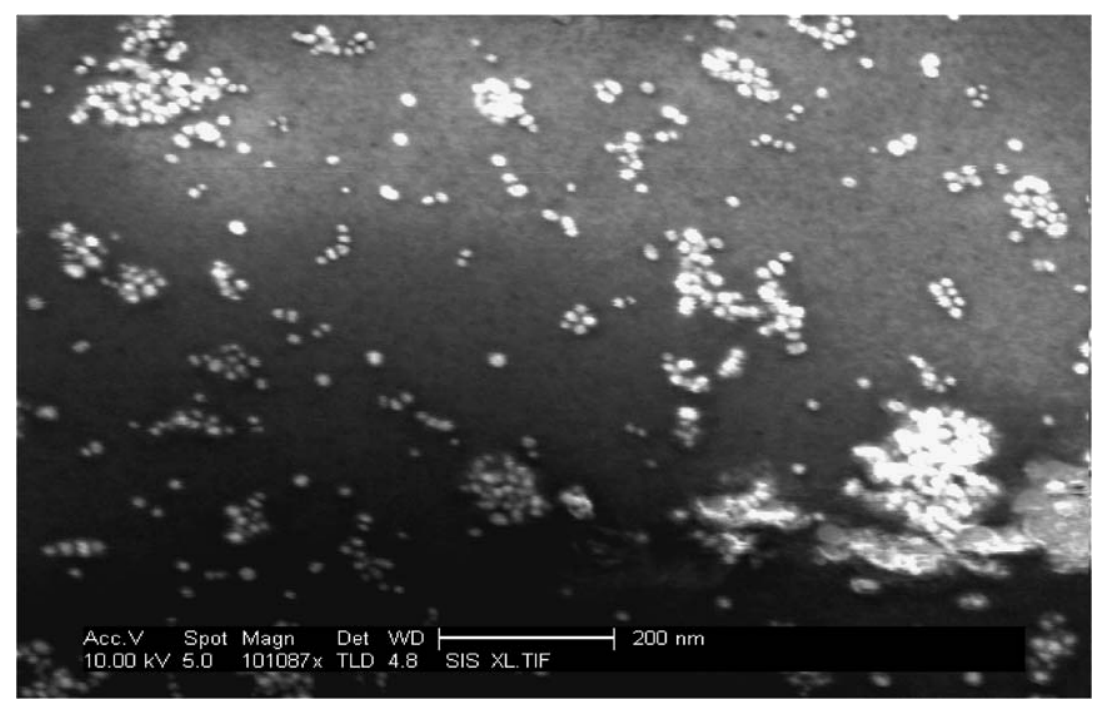

Fig. 3. SEM image of an Ag colloidal solution.

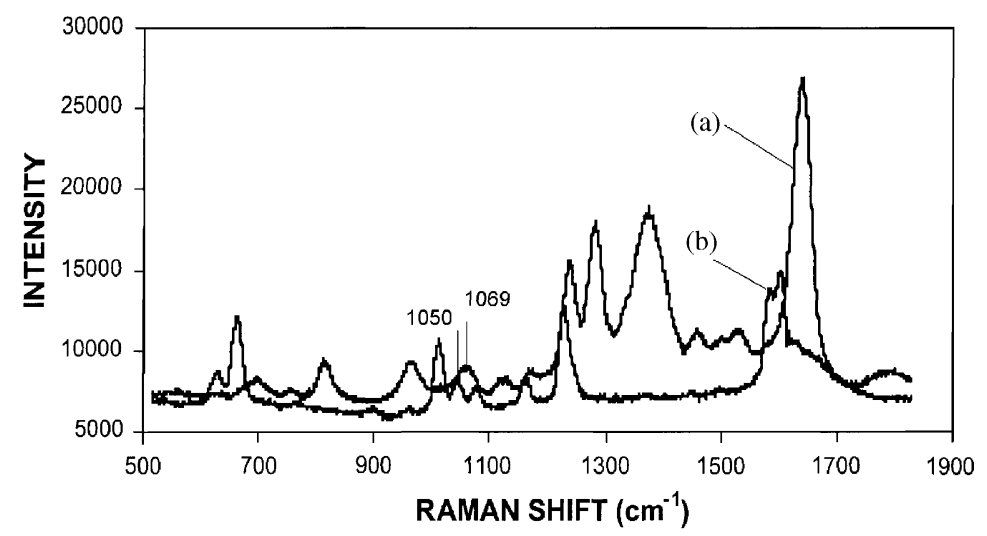

Fig. 4. Enhancement measurement for pyridine. The upper (SERS) spectrum is for a $10^{-9} \mathrm{M}$ solution in a silver colloid suspension ( $\mathrm{Ag} \sim 2 \times 10^{-4} \mathrm{M}$ ), and the lower (standard Raman) spectrum is for a $1 \mathrm{M}$ pyridine solution in the absence of colloid.

particle diameter is $\sim 30 \mathrm{~nm}$. The absorption maxima were found to be at 396-400 $\mathrm{nm}$ from the UV-VIS studies.

Our first studies using SERS were with pyridine to establish that our colloidal solution was reproducible, to benchmark our optical system, and to determine the maximum enhancement. Many authors have reported SERS using Ag colloidal solutions mixed with pyridine and its derivatives (Creighton et al., 1979; Takenaka, Eda, Mabuchi, Fujiyoshi, \& Uyeda, 1984). Our results show that the concentration ratio of pyridine to Ag colloid significantly affects the enhancement. In Fig. 4 we show the maximum enhancement that we measured for any pyridine: $\mathrm{Ag}$ colloid concentration ratio. The upper spectrum is for a $10^{-9} \mathrm{M}$ pyridine solution in a silver colloid suspension $\left(\mathrm{Ag} \sim 2 \times 10^{-4} \mathrm{M}\right)$, and the lower curve is for a $1 \mathrm{M}$ pyridine solution in the absence of colloid. The enhancement $\left(\sim 10^{9}\right)$ was determined by comparing 
the SERS peak at $1069 \mathrm{~cm}^{-1}$ with the Raman peak at $1050 \mathrm{~cm}^{-1}$ in the normal Raman spectrum of pyridine (1 M). The enhanced Raman spectrum with silver colloid shows some new structures as well as some peak shifting. Both samples had the same laser power and exposure time.

Experiments with MC4100 E. coli (ATCC 35695), Pseudomonas aeruginosa (ATCC 47085), and LT2 Salmonella (ATCC 19585) bacteria were then performed to explore the parameters involved with detecting bacteria with SERS. Bacterial samples were grown till the stationary growth stage after incubating for $17 \mathrm{~h}$ in Luria broth (LB) growth medium. The bacterial concentrations were in the range of $10^{7}-10^{8} \mathrm{cfu} / \mathrm{ml}$. Then the samples were centrifuged and washed twice before re-suspending them in de-ionized water.

We also obtained SERS spectra for $N$-acetyl glucosamine, L-lysine, D-alanine and D-glutamic acid to provide the background to interpret the Raman spectra of bacteria. The $N$-acetyl glucosamine, L-lysine, D-alanine and D-glutamic acid, silver nitrate (99.9999\%), and sodium borohydride were obtained from Aldrich Chemical Co. Pyridine (99.9\%) was supplied by Fisher Scientific.

\section{Results}

To distinguish microorganisms, we need to identify or characterize the chemical groups present in their cell walls. This outer wall is responsible for their pathogenic properties. The cell wall composition also varies for different strains such as gram-positive and gram-negative species. The dominant components of the cell wall are amino acids and polysaccharides. We obtained SERS spectra of three main amino acids that are present in a bacterial cell wall to establish their Raman signatures. Fig. 5 shows SERS spectra of L-lysine, D-glutamic acid and D-alanine, and the spectra are seen to be very similar. The characteristic amino acid peaks shown in the figure agree with those reported by Dou, Jung, Yamamoto, Doi, and Ozaki (1999). The presence of the main band at $1370-1375 \mathrm{~cm}^{-1}$ indicates the strong interaction between carboxylate groups in amino acids with silver. We also investigated $N$-acetyl glucosamine (NAG), an important constituent molecule in the peptidoglycan layer in the cell wall, and the NAG SERS spectrum shown in Fig. 6 is similar to the spectra for the amino acids.

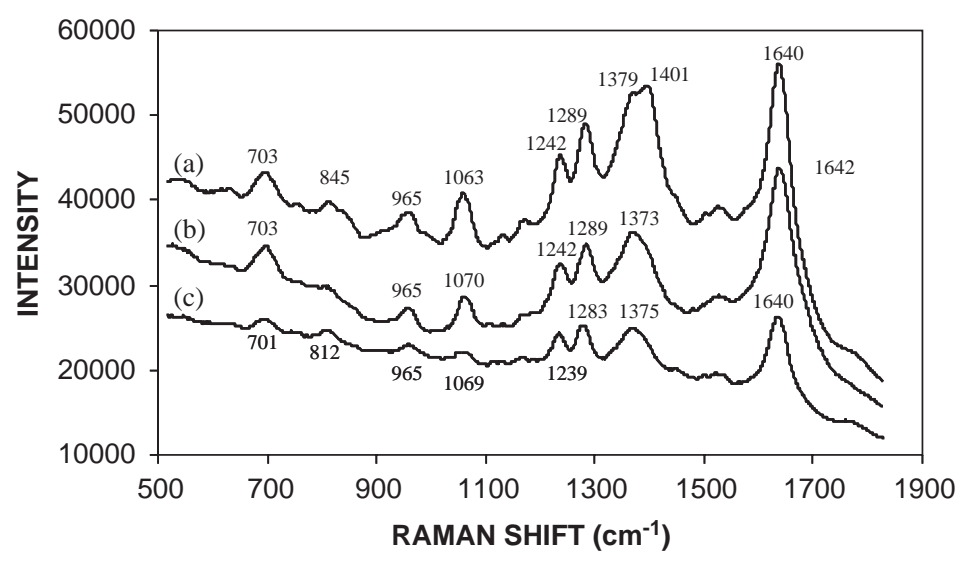

Fig. 5. SERS spectra of amino acids in Ag colloidal solution: (a) D-glutamic acid $(0.2 \mu \mathrm{M})$, (b) D-alanine (1 $\mu \mathrm{M})$, (c) L-lysine $(1 \mu \mathrm{M})$. The intensities have been rescaled to prevent overlapping of these spectra. 


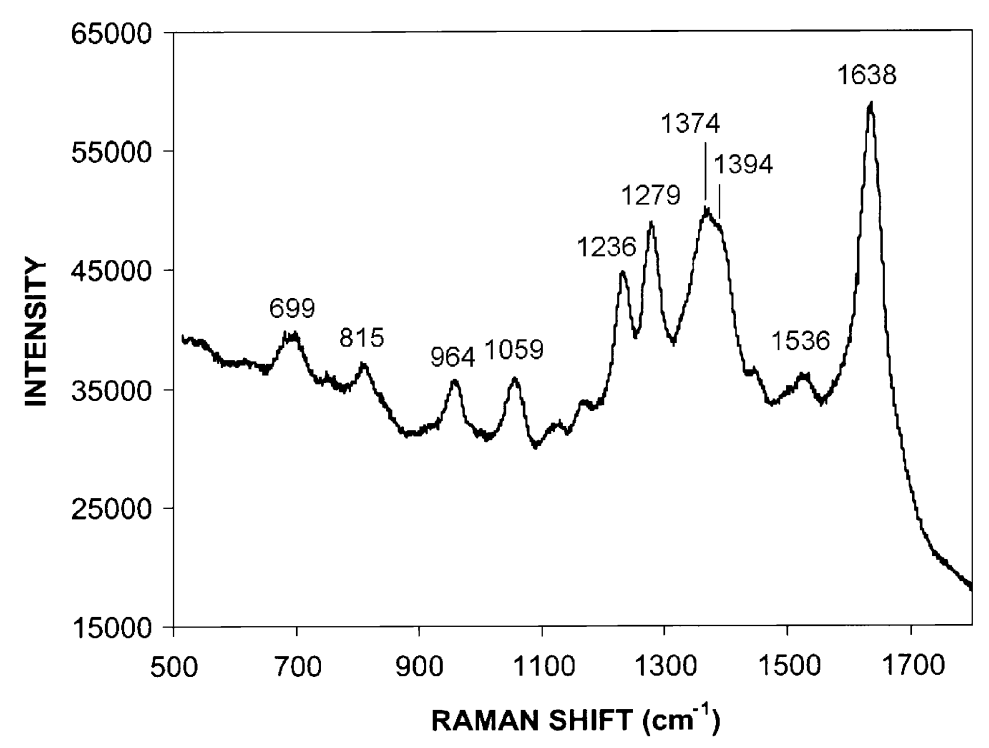

Fig. 6. SERS spectrum of $N$-acetyl glucosamine $(1 \mu \mathrm{M})$ in Ag colloid.

Conventional Raman scattering from a bacterial dispersion does not yield a useful Raman fingerprint. This is shown in Fig. 7(a), a spectrum obtained for E. coli bacteria grown in LB growth medium and injected into the cuvette without silver colloid. The spectrum is dominated by a broad fluorescence band that obscures the Raman signal. Fig. 7(b) is the Raman spectrum of the same bacterial solution diluted by a factor of $10^{4}$ with de-ionized water. There is still a fluorescent background in the region $500-1000 \mathrm{~cm}^{-1}$ and a characteristic water peak at $1640 \mathrm{~cm}^{-1}$. The broad fluorescence band is reduced, but there are no characteristic Raman peaks. When the bacterial suspension was washed and centrifuged, then diluted by a factor of 50 with the silver colloid, we obtained the SERS spectrum shown in Fig. 8.

By means of SERS the Raman peaks are substantially enhanced and fluorescence is quenched. Figs. 8-10 present SERS spectra of three different bacterial species that we investigated, E. coli, Salmonella typhimurium, and Pseudomonas aeruginosa, respectively. The volume ratios of bacterial suspension in DI water to colloidal suspension are reported as $V_{\mathrm{B}} / V_{\mathrm{C}}$ in the figure captions. The spectra are quite similar and have many peaks in common. Major peaks at $1355-1360 \mathrm{~cm}^{-1}, 1287-1292 \mathrm{~cm}^{-1}, 1240-1242 \mathrm{~cm}^{-1}$, $1175 \mathrm{~cm}^{-1}$, and $1540 \mathrm{~cm}^{-1}$ are the characteristic amino acid peaks seen in Figs. 5 and 6 . Peaks in the region $500-1100 \mathrm{~cm}^{-1}$ are due to the various other groups that are present in the bacterial cell (Schuster, Reese, Urlaub, Gapes, \& Lendl, 2000). These include glycosides, nucleic acids (cytidine, uracil), proteins (tyrosine), phenylalanine, carbohydrates and unsaturated fatty acid and lipids. The peak at $1640 \mathrm{~cm}^{-1}$ is the amide peak and it overlays the water peak.

Although the spectra in Figs. 8-10 are similar, there are some differences in the region $500-1100 \mathrm{~cm}^{-1}$, and some differences in the major bands in the region $1300-1500 \mathrm{~cm}^{-1}$. Fig. 10 presents two spectra for PAO1 Pseudomonas aeruginosa obtained on different days using different batches of the colloid. The reproducibility is good, but there are differences in the region $1300-1500 \mathrm{~cm}^{-1}$ where there are major bands. This region can be expected to involve the groups that have the strongest interaction with the silver surface. It is likely that slight variations in the parameters such as the concentration of bacteria, the concentration of $\mathrm{Ag}$, and the time of adsorption affect this interaction and alter the spectrum. 

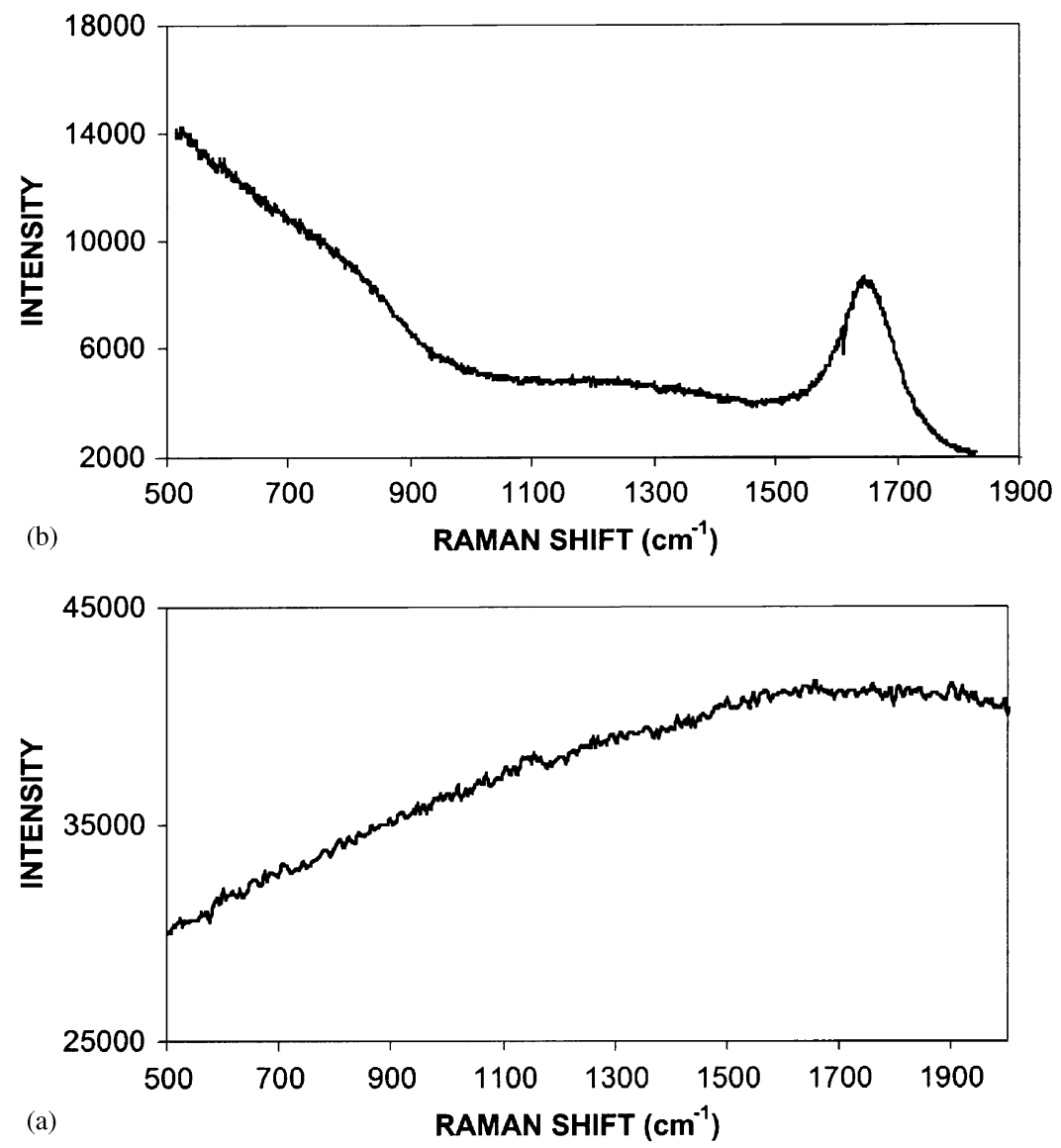

Fig. 7. Raman spectrum of (a) concentrated Escherichia coli bacteria in LB growth medium, and (b) the Raman spectrum after diluting the concentrated bacterial suspension by a factor of $10^{4}$ with de-ionized water.

Fig. 11 compares spectra of the three different bacterial samples in the region $500-1100 \mathrm{~cm}^{-1}$. Slight differences in the spectra may be attributed to the structural differences in the cell walls. Ivanova et al. (2000) found that in marine-water bacteria the genus characteristic patterns of phospholipids and fatty acids are useful in genera discrimination. Fig. 11 shows some differences in the spectra in the regions $550-650 \mathrm{~cm}^{-1}$, $\sim 750 \mathrm{~cm}^{-1}$, and $900-1000 \mathrm{~cm}^{-1}$. To display the differences, Fig. 12 shows spectra constructed by taking the MC4100 E. coli spectrum as reference. The spectra of (d) MC4100 E. coli, (a) P. aeruginosa, (b) BL21 E. coli, and (c) LT2 Salmonella were normalized to yield the same intensity at $754 \mathrm{~cm}^{-1}$. The intensity of the MC4100 E. coli spectrum was subtracted from those of the other spectra at each wave number to generate the difference plot. Consequently, the straight line at zero intensity is the reference MC4100 E. coli spectrum. The plot clearly shows that there is very little difference between the two different strains of $E$. coli, but some differences can be observed for Salmonella and appreciable differences for Pseudomonas. A more rigorous treatment of the data using, say, principle component analysis would be a useful alternate approach. 


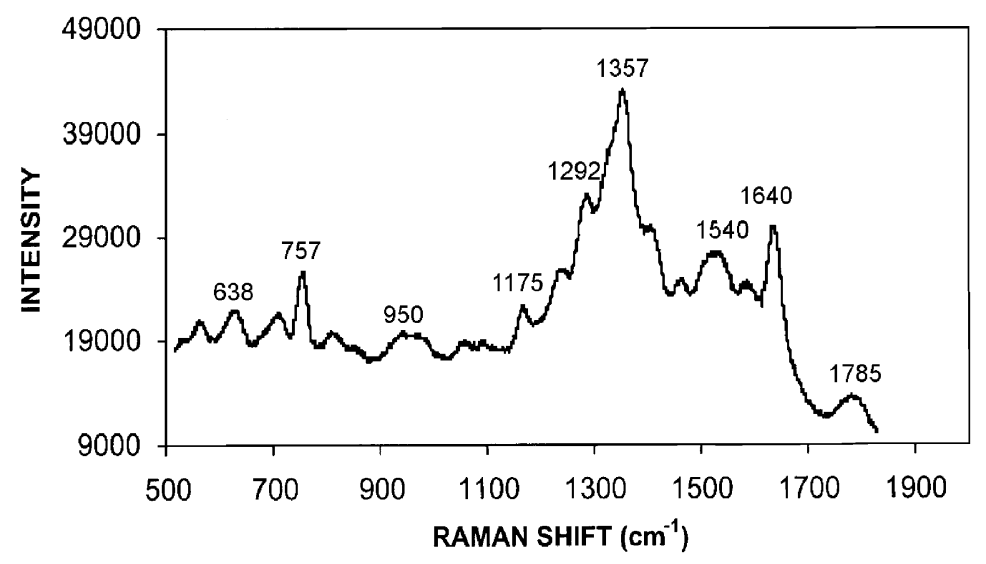

Fig. 8. SERS spectrum of BL21 Escherichia coli for $V_{\mathrm{B}} / V_{\mathrm{C}}=1 / 50$.

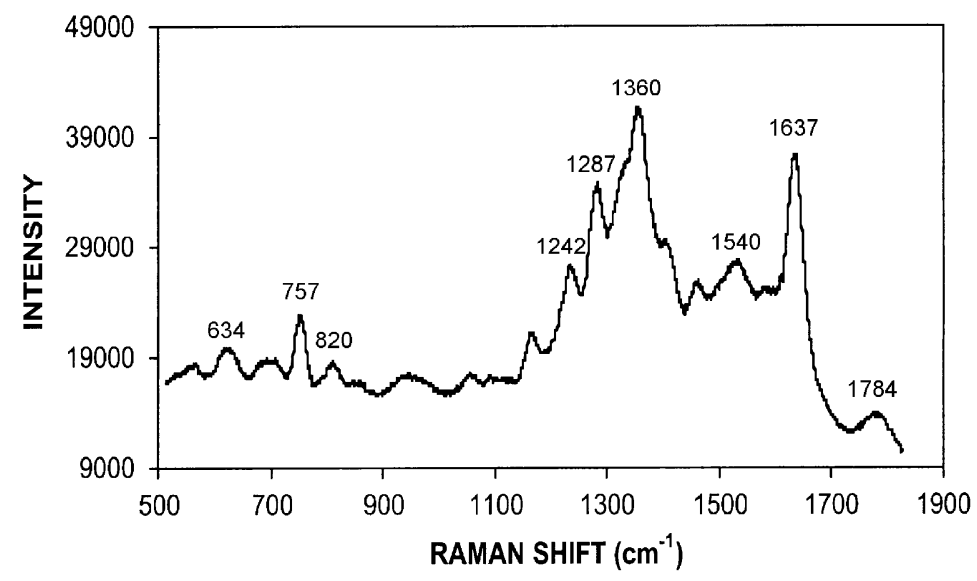

Fig. 9. SERS spectrum of LT2 Salmonella typhimurium for $V_{\mathrm{B}} / V_{\mathrm{C}}=1 / 50$.

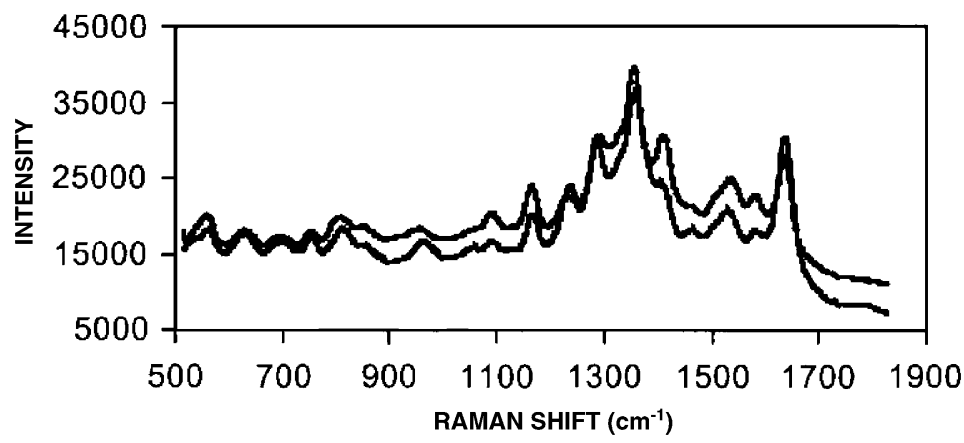

Fig. 10. SERS spectra of PAO1 Pseudomonas aeruginosa for $V_{\mathrm{B}} / V_{\mathrm{C}}=1 / 100$ obtained with two different batches of colloids. 


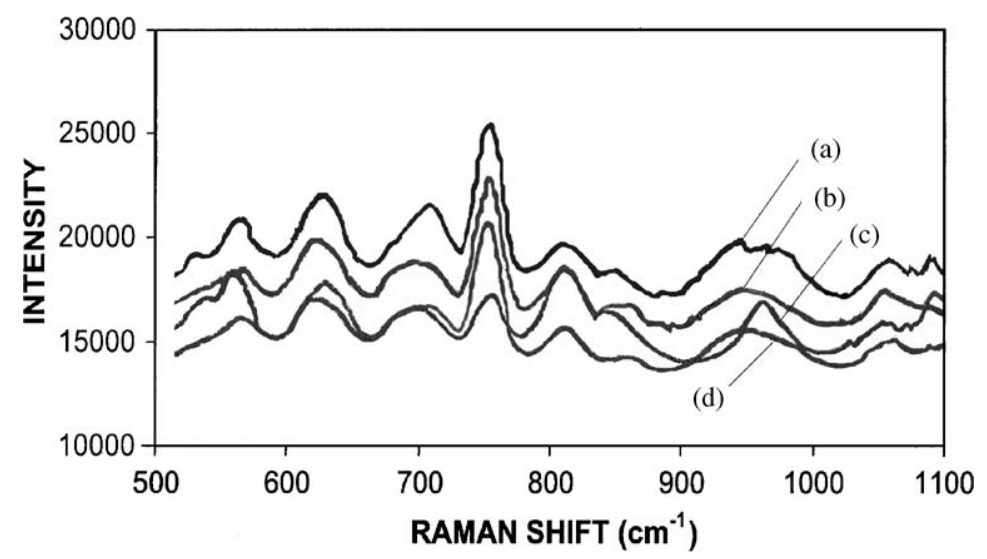

Fig. 11. SERS spectra in the region $500-1100 \mathrm{~cm}^{-1}$ for bacteria: (a) LT2 Salmonella typhimurium $\left(V_{\mathrm{B}} / V_{\mathrm{C}}=1 / 50\right)$, (b) BL21 Escherichia coli $\left(V_{\mathrm{B}} / V_{\mathrm{C}}=1 / 50\right)$, (c) PAO1 Pseudomonas aeruginosa $\left(V_{\mathrm{B}} / V_{\mathrm{C}}=1 / 100\right)$, (d) MC4100 Escherichia coli $\left(V_{\mathrm{B}} / V_{\mathrm{C}}=1 / 50\right)$.

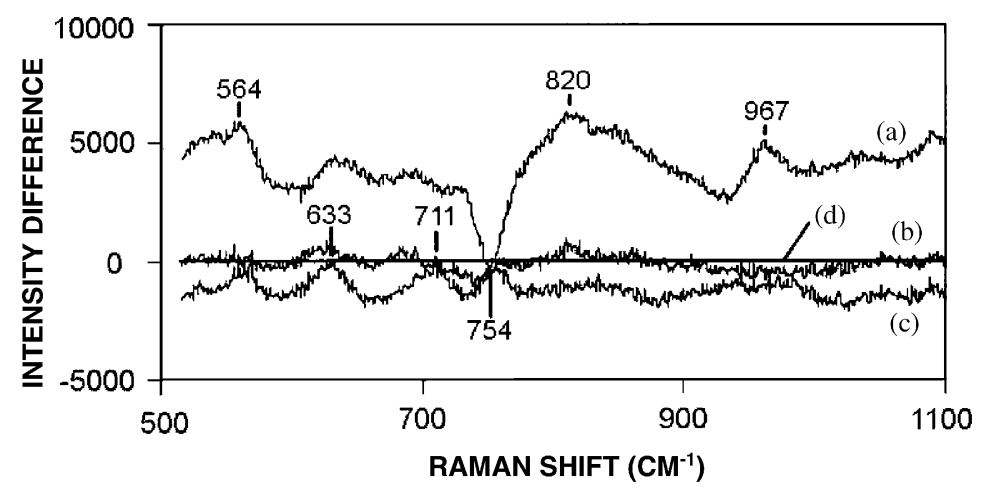

Fig. 12. A plot of the intensity difference between reference spectrum (d) MC4100 Escherichia coli and (a) PA01 Pseudomonas aeruginosa, (b) BL21 Escherichia coli, and (c) LT2 Salmonella typhimurium.

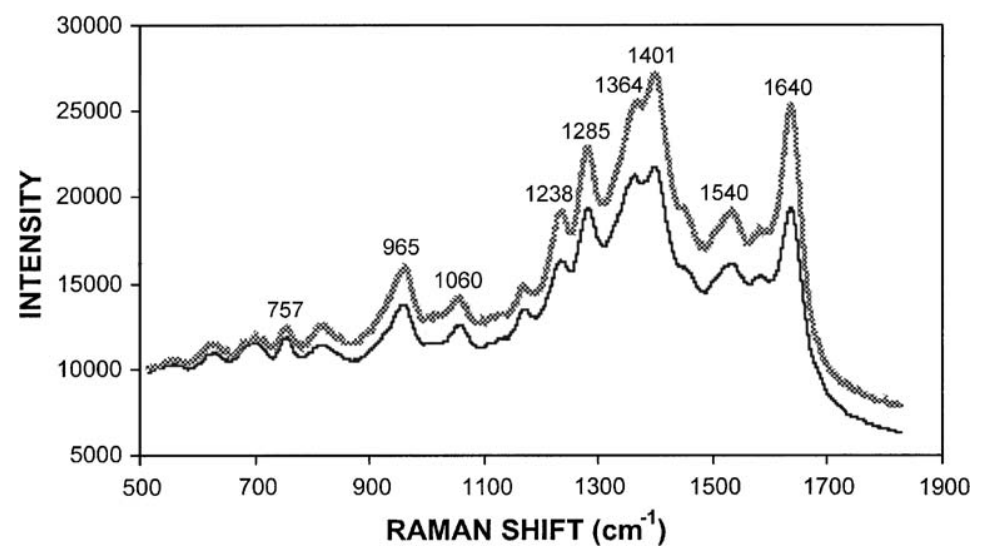

Fig. 13. SERS spectra of two samples of aerosolized ML4100 E. coli. 


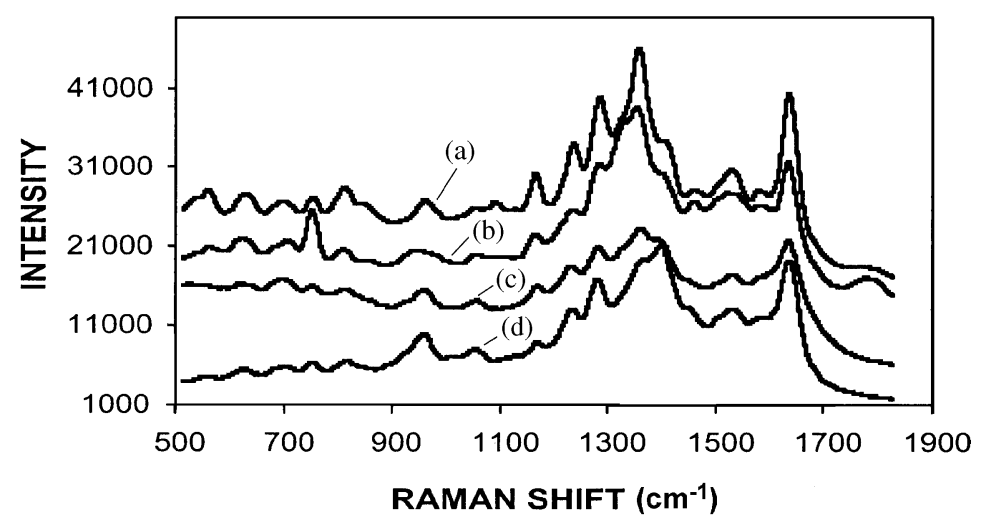

Fig. 14. SERS spectra of (a) non-aerosolized PAO1 Pseudomonas aeruginosa (b) non-aerosolized ML4100 Escherichia coli (c) aerosolized PAO1 Pseudomonas aeruginosa and (d) aerosolized ML4100 Escherichia coli. The intensities have been rescaled to prevent overlapping of these spectra.

All of the results shown above were done with bacteria samples taken from stock solutions, but the primary objective of the work was to obtain SERS spectra of airborne bacteria. Samples were nebulized, impacted, and collected as indicated above. Fig. 13 shows spectra from two identical samples of aerosolized $E$. coli to test the reproducibility of the method, and Fig. 14 compares SERS spectra for aerosolized and non-aerosolized E. coli and Pseudomonas. The spectra of the aerosolized bacteria are nearly the same as those for the stock suspensions.

\section{Comments}

We have demonstrated that the bioaerosols studied can be characterized by SERS. The method is relatively simple and fast compared with conventional methods. We did not characterize the aerosol with respect to the size distribution, number density, and other aerosol properties. Consequently, we are not able to establish the sensitivity of the method based on this work, but it is clear that the sensitivity and detection limits depend on the ratio of the number density of bacteria to the number density of the colloidal particles as well as the size and surface properties of the colloid particles.

The collection efficiency of the impactor/collector was not evaluated in this study, but we attempted to reproduce the collection by using the same flow rate and nebulization time for each experiment with aerosolized bacteria.

\section{Acknowledgements}

The authors would like to thank Professors Daniel T. Schwartz and François Baneyx for their interest and advice. The contributions of Mirna Mujacic in Professor Baneyx's laboratory are highly appreciated. She provided the bacterial samples. The authors would also like to thank the National Science Foundation for Grant Number CTS-9982413, the University of Washington/PNNL Joint Institute for Nanotechnology, and the University of Washington Center for Nanotechnology for their financial support. 


\section{References}

Aizenberg, V., Reponen, T., Grinshpun, S. A., \& Willeke, K. (2000). Performance of the Air-O-Cell, Burkard, and Button samplers for total enumeration of airborne spores. American Industrial Hygiene Association Journal, 61, 855-864.

Alexander, T. A., Pellegrinol, P. M., \& Gillespie, J. B. (2003). Near-infrared surface-enhanced Raman scattering-mediated detection of single optically trapped bacterial spores. Applied Spectroscopy, 57, 1340-1345.

Carey, P. R. (1982). Biochemical applications of Raman and resonance Raman spectroscopies. New York: Academic Press.

Creighton, J. A., Blatchford, C. G., \& Albrecht, M. G. (1979). Plasmon resonance enhancement of Raman scattering by pyridine adsorbed on silver or gold sol particles of size comparable to the excitation wavelength. Faraday Transactions II, 75, 790798.

Dou, X., Jung, Y. M., Yamamoto, H., Doi, S., \& Ozaki, Y. (1999). Near-infrared excited surface-enhanced Raman scattering of biological molecules on gold colloid I: effects of $\mathrm{pH}$ of the solutions of amino acids and of their polymerization. Applied Spectroscopy, 53, 133-138.

Efrima, S., \& Bronk, B. V. (1998). Silver colloids impregnating or coating bacteria. Journal of Physical Chemistry B, 102,5947 -5950 .

Freemantle, M. (2003). An incisive probe for biomolecules. Chemical and Engineering News, pp. 36-39, 13 January.

Hairston, P. P., Ho, J., \& Quant, F. R. (1997). Design of an instrument for real-time detection of bioaerosols using simultaneous measurement of particle aerodynamic size and intrinsic fluorescence. Journal of Aerosol Science, 28, 471-482.

Henningson, E. W., Lundquist, M., Larsson, E., Sandstrom, G., \& Forsman, M. (1997). A comparative study of different methods to determine the total number and survival ratio of bacteria in aerobiological samplers. Journal of Aerosol Science, 28, 459469.

Hill, S. C., Pinnick, R. G., Nachman, P., Chen, G., Chang, R. K., Mayo, M. W., \& Fernandez, G. L. (1995). Aerosol-fluorescence spectrum analyzer: real-time measurement of emission spectra of airborne biological particles. Applied Optics, 34, 71497155 .

Ivanova, E. P., Zhukova, N. V., Svetashev, V. I., Gorshikova, N. M., Kurilenko, V. V., Frolova, G. M., \& Mikhailov, V. V. (2000). Evaluation of phospholipid and fatty acid compositions as chemotaxonomic markers of Alteromonas-like Proteobacteria. Current Microbiology, 41, 341-345.

Jarvis, R. M., \& Goodacre, R. (2004). Discrimination of bacteria using surface-enhanced Raman spectroscopy. Analytical Chemistry, 76, 40-47.

Keir, R., Sadler, D., \& Smith, W. E. (2002). Preparation of stable, reproducible silver colloids for us as surface-enhanced resonance Raman scattering substrates. Applied Spectroscopy, 56, 551-559.

Laucks, M. L., Davis, E. J., Roll, G., \& Schweiger, G. (2000). Raman characterization of bioaerosols—pollen. Journal of Aerosol Science, 31, 307-319.

Lee, B. U., \& Kim, S. S. (2002). Sampling E. coli and B. subtilis bacteria bioaerosols by a new type of impactor with a cooled impaction plate. Journal of Aerosol Science, 34, 1097-1100.

Lin, X., Reponen, T., Willeke, K., Wang, Z., Grinshpun, S. A., \& Trunov, M. (2000). Survival of airborne microorganisms during swirling aerosol collection. Aerosol Science and Technology, 32, 184-196.

Mainelis, G., Adhikari, A., Willeke, K., Lee, S. -A., Reponen, T., \& Grinshpun, S. A. (2002a). Collection of airborne microorganisms by a new electrostatic precipitator. Journal of Aerosol Science, 33, 1417-1432.

Mainelis, G., Górny, R. L., Reponen, T., Trunov, M., Grinshpun, S. A., Baron, P., Yadav, J., \& Willeke, K. (2002b). Effect of electrical charges and fields on injury and viability of airborne bacteria. Biotechnology and Bioengineering, 79, $229-241$.

Mainelis, G., Grinshpun, S. A., Willeke, K., Reponen, T., Ulevicius, V., \& Hintz, P. J. (1999). Collection of airborne microorganisms by electrostatic precipitation. Aerosol Science and Technology, 30, 127-144.

Mainelis, G., Willeke, K., Adhikari, A., Reponen, T., \& Grinshpun, S. A. (2002). Design and collection efficiency of a new electrostatic precipitator for bioaerosol collection. Aerosol Science and Technology, 36, 1073-1085.

Mainelis, G., Willeke, K., Baron, P., Reponen, T., Grinshpun, S. A., Górny, R. L., \& Trakumas, S. (2001). Electrical charges on airborne microorganisms. Journal of Aerosol Science, 32, 1087-1110.

Marijnissen, J. C. M., Van Wuijckhuijse, A. L., \& Stowers, M. A. (2003). The latest development in the aerosol laser time-of-flight mass spectrometer for the chemical analysis of aerosol particles on-line, with an emphasis on bio-aerosols. Paper 120B2, 22nd Annual AAAR Conference, Anaheim, CA, October 20-24.

Murray, K., Jackson, S., Mishra, S., \& Kim, J.-K. (2003). Laser desorption mass spectrometry for bioaerosol detection. Paper 10B3, 22nd Annual AAAR Conference, Anaheim, CA, October 20-24. 
Nachma, P., Chen, G., Pinnick, R. G., Hill, S. C., Chang, R. K., Mayo, M. W., \& Fernandez, G. L. (1996). Conditional-sampling spectrograph detection system for fluorescence measurements of individual airborne biological particles. Applied Optics, 35, 1069-1076.

Parker, F. S. (1983). Applications of infrared, Raman, and resonance Raman spectroscopy in biochemistry. New York: Plenum Press.

Schuster, K. C., Reese, I., Urlaub, E., Gapes, J. R., \& Lendl, B. (2000). Multidimensional information on the chemical composition of single bacterial cells by confocal Raman microspectroscopy. Analytical Chemistry, 72, 5529-5534.

Stewart, S. L., Grinshpun, S. A., Willeke, K., Terzieva, S., Ulevicius, V., \& Donnelly, J. (1995). Effect of impact stress on microbial recovery on an agar surface. Applied Environmental Microbiology, 61, 1232-1239.

Takenaka, T., Eda, K., Mabuchi, M., Fujiyoshi, Y., \& Uyeda, N. (1984). Surface enhanced Raman scattering by isomeric monobromopyridines adsorbed on gold and silver sol particles. Bulletin of the Institute for Chemical Research, Kyoto University, 62, 219-232.

Walter, K. (1998). Science \& technology review (pp. 4-9). Lawrence Livermore National Laboratory.

Wang, Z., Reponen, T., Grinshpun, S. A., Górny, R. L., \& Willeke, K. (2001). Effect of sampling time and air humidity on the bioefficiency of filter samplers for bioaerosol collection. Journal of Aerosol Science, 32, 661-674.

Wang, Z., Reponen, T., Willeke, K., \& Grinshpun, S. A. (1999). Survival of bacteria on respirator filters. Aerosol Science and Technology, 30, 300-308. 